
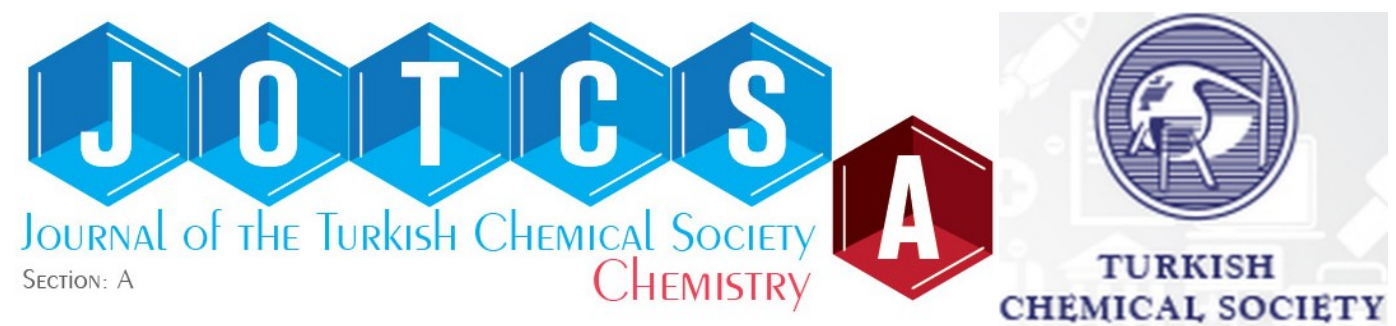

\title{
Some Properties of an Unmetalled Phthalocyanine Obtained by Chance at Low Temperature
}

\author{
Mevlude Canlica ${ }^{1 *}$ iD \\ $1 *$ Yildiz Technical University, Faculty of Science and Arts, Department of Chemistry, Davutpasa Campus, \\ 34220 Esenler, Istanbul, Turkey.
}

\begin{tabular}{l}
\hline Abstract: A novel tetrasubstituted metal-free phthalocyanine bearing phthalonitrile groups on the \\
peripheral positions was synthesized accidentally by the cyclotetramerization reaction of the $4,4^{\prime}-(1,3-$ \\
phenylenebis $($ oxy) $)$ diphthalonitrile. For photodynamic therapy (PDT) application, photophysical \\
properties including fluorescence quantum yield $\left(\Phi_{\mathrm{F}}\right)$, fluorescence lifetime $\left(\mathrm{T}_{\mathrm{F}}\right)$, triplet state lifetime \\
$(\mathrm{TT})$, and triplet quantum yield $\left(\Phi_{\mathrm{T}}\right)$ and photochemical properties including singlet oxygen quantum \\
yield $\left(\Phi_{-}\right)$and photodegradation quantum yield $\left(\Phi_{\mathrm{d}}\right)$ as well as magnetic circular dichroism (MCD) \\
properties were described. The $\Phi_{T}$ of the phthalocyanine was 0.81 . The obtained lifetime $(\mathrm{TT})$ was $40 \mu \mathrm{s}$ \\
for metal-free PC.
\end{tabular}

Keywords: Metal-free phthalocyanine, singlet oxygen quantum yield, magnetic circular dichroism.

Submitted: . October 07, 2020. Accepted: October 13, 2020.

Cite this: Canlıca M. Some Properties of an Unmetalled phthalocyanine Obtained by Chance at Low Temperature. JOTCSA. 2020;7(3):875-82.

DOI: https://doi.org/10.18596/jotcsa.807291.

*Corresponding author. E-mail: (mcanlica@yahoo.com), Tel: (+902123834161).

\section{INTRODUCTION}

Phthalocyanines (Pcs) are macrocyclic compounds that consist of 4 pyrrolic subunits. The first synthesis of a Pc was found by accident in 1907, and it involves with low yield the reaction of ocyanobenzamide in refluxing ethanol (1). However, nowadays a substituted phthalonitrile can be easily prepared by many different routes. Substituted Pcs can be formed from these phthalonitriles. The most commonly used method for the synthesis of $\mathrm{Pc}$ is cyclotetramerization of the phthalonitrile to form a Pc. A Pc offers 16 possible sites for peripheral and non-peripheral substitutions and a central cavity with metal ions and this makes them useful compounds in different fields and them are applied as dye, catalysis in a wide range of areas $(2,3)$ non-linear optics (4), Langmuir-Blodgett thin films $(5,6)$, chemical sensors $(7)$, as photosensitizers in PDT (8-11) and as photocatalysts (12-14). On the other hand, ball-type structures of Pcs were first obtained as a new class of compound by Zefirova in $2002(15,16)$ and both Pcs and their ball-type derivatives (BPCS) have gained significance in a number of fields including their use as photosensitizers in photodynamic therapy (PDT) of cancer (17-20). In this study, a metal-free phthalocyanine, which was obtained accidentally during a reaction between 4-nitrophthalonitrile and resorcinol (1,3-dihydroxybenzene), was investigated photo-physico-chemical behaviors and MCD properties.

\section{EXPERIMENTAL SECTION}

\section{Materials and methods}

All equipments and chemicals that were used during all experimental and measurements are the same as the paper published earlier in the literature. The photophysical and photochemical properties of metal-free Pc were performed according to methods previously described in the literature $(19,20)$. 


\section{Synthesis}

Synthesis of 2(3), 9(10), 16(17), 23(24)-tetrakis(4-(3-oxyphenoxy) phthalocyanine (5):

Compound 2 (1.272 g, $11.54 \mathrm{mmol}$ ) was dissolved in dry DMSO (25 mL) and compound 1 (3.98 g, $23.08 \mathrm{mmoL}$ ) was added under inert atmosphere. To this reaction mixture finely ground anhydrous potassium carbonate $(4.00 \mathrm{~g}, 28.94 \mathrm{mmol})$ was added. The color of the mixture changed to the green from light brown while it was continued stirring at the end of 5 days at $70{ }^{\circ} \mathrm{C}$. after a total of 6 days of stirring at this temperature, the entire green reaction mixture was then poured onto cracked ice to produce a precipitate, and the precipitate was washed sequentially with ethanol and methanol resulting in the formation of a pure green precipitate. Finally, it was purified by silica gel column chromatography using gradient of chloroform, tetrahydrofuran as eluent and metalfree Pc was obtained as a green powder which has $\mathrm{mp}>350{ }^{\circ} \mathrm{C}$ and is soluble in THF, DMSO, and DMF. Yield $0.069 \mathrm{~g}$. UV-Vis (DMSO): $\lambda_{\max } / \mathrm{nm}: 700$,

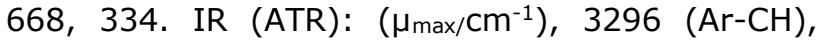
$2238(\mathrm{CN}), 1717(\mathrm{CO}), 1596(\mathrm{C}=\mathrm{C}), 1121 / 1083$ $(\mathrm{C}-\mathrm{O}-\mathrm{C}) .{ }^{1} \mathrm{H}-\mathrm{NMR}\left(\mathrm{DMSO}-d_{6}\right): \delta, \mathrm{ppm}: 7.85-7.00$ $(40 \mathrm{H}, \mathrm{Ar}-\mathrm{H})$. Anal. calcd. for $\mathrm{C}_{88} \mathrm{H}_{42} \mathrm{~N}_{16} \mathrm{O}_{8}$ : C, 72.82; H, 2.92; N, 15.44, found: C, 72.79; H, $2.88 ; \mathrm{N}, 15.45 \%$. MALDI-TOF-MS: $\mathrm{m} / \mathrm{z}$ calcd. 1451.4 , found $1453.5[\mathrm{M}+2 \mathrm{H}]^{+}$.

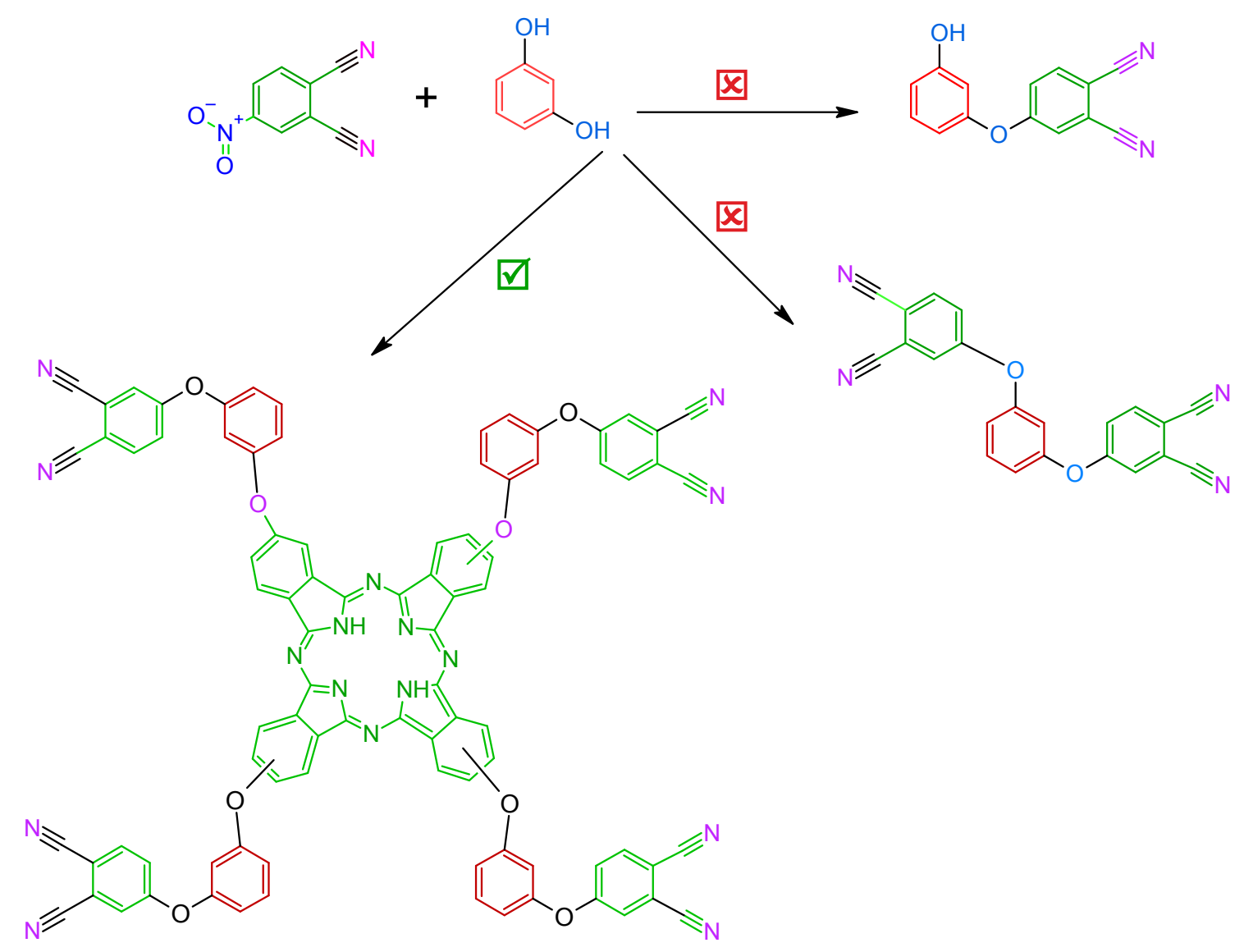

Scheme 1. Synthetic pathway for metal-free phthalocyanine (5) i: DMSO, 6 days, $\mathrm{K}_{2} \mathrm{CO}_{3}$.

\section{RESULTS AND DISCUSSION}

\section{Synthesis and spectroscopic characterization} A reaction of 4,4'-(1,3phenylenebis(oxy))diphthalonitrile (3) was started for the synthesis of the one which was intended for a BPc (Scheme 1). At the end of the fifth day, a green color was observed in the reaction tube. Metal-free phthalocyanine was obtained accidentally during a reaction which it give two possible products ( 3 and $\mathbf{4}$ ) between 4- nitrophthalonitrile and resorcinol at low temperature. This showed that compound $\mathbf{3}$ gave quickly metal-free Pc (5) by self-condensation; it means that compound $\mathbf{5}$ occurred unexpectedly under the experimental conditions (21). So, compound 3 was not isolated and characterized (22).

After purification, the novel tetrakis (4-(3oxyphenoxy) substituted Pc (5) was characterized by a handful of common spectroscopic techniques, 
including FTIR, ${ }^{1} \mathrm{H}$ NMR, MALDI-TOF MS and UVVis, along with elemental analysis.

FT-IR spectrum of the $\mathrm{H}_{2} \mathrm{Pc}$ was obtained with ATR and the spectrum showed $-\mathrm{C} \equiv \mathrm{N}$ stretching at 2238 $\mathrm{cm}^{-1}$. this value confirmed that compound $\mathbf{5}$ contains cyano end groups as determined by FT-IR spectroscopy. Aromatic C-H at $3296 \mathrm{~cm}^{-1}$, aromatic $\mathrm{C}=\mathrm{C} 1596 \mathrm{~cm}^{-1}$ and $\mathrm{C}-\mathrm{O}-\mathrm{C}$ vibrations at $1121 / 1083 \mathrm{~cm}^{-1}$ were observed. The ${ }^{1} \mathrm{H}$ NMR spectrum of (5) showed a multiplicity between 7.85 - $7.00 \mathrm{ppm}$ in deuterated DMSO as the solvent. The inner core protons could not be observed. This may be due to the strong aggregation between the Pc rings. MALDI-TOF result showed the molecular peak of the compound 5 at $\mathrm{m} / \mathrm{z}$ 1292.57, and also elemental analysis supported compound $\mathbf{5}$.

The electronic absorption spectra for Pcs is generally characterized by two bands. One of both, the $\mathrm{Q}$ band is found in the longer wavelengths around $600-700 \mathrm{~nm}$ region were responsible for the green color of this complex. The other that are referred to as the $B$ (Soret band). Figure 1 show the typical strong absorption bands. These transitions were assigned to $\Pi-\Pi^{*}$ transition between bonding and antibonding molecular orbitals.In the case of metal-free phthalocyanine, this band generally split into at two components. The $B$ band is located at the shorter wavelengths around $300-400 \mathrm{~nm}$ region. B band arises mainly from the $\Pi$ to $\Pi^{*}$ transition. The $Q$ band results from $\Pi \rightarrow \Pi^{*}$ transition that involves $a_{1 \mathrm{u}}$ to $\mathrm{e}_{\mathrm{g}}$ and the Soret band result from the $\Pi \rightarrow \Pi^{*}$ transition that involves $a_{2 u}$ or $b_{2 u}$ to $e_{g}$. The non-degenerated $\mathrm{e}_{\mathrm{g}}$ level leads to the observed split $\mathrm{Q}$ band in the UV-vis spectra for $\mathrm{H}_{2} \mathrm{Pc}$. On the other word, the splitting of the $\mathrm{Q}$ band is due to the splitting in the $\mathrm{e}_{\mathrm{g}}$ level. In the spectrum of compound $\mathbf{5}$, a loss was observed in the geometrically induced splitting between the $Q_{x}$ and $Q_{y}$ components (23).

Table 1: Photophysical and photochemical data of the PC.

\begin{tabular}{|c|c|c|c|c|c|c|c|c|c|c|}
\hline Compound & AAbs & $\lambda E m s$ & $\lambda E x c$ & $\lambda$ Stokes & $\Phi_{F}$ & $\Phi_{\mathbf{T}}$ & $\Phi_{\Delta}$ & $\mathbf{T}_{\mathrm{T}}(\mathbf{n s})$ & $\mathbf{T}_{\mathrm{T}}(\boldsymbol{\mu} \boldsymbol{S})$ & $\Phi_{\mathrm{d}}\left(\times 10^{-6}\right)$ \\
\hline $\mathrm{H}_{2} \mathbf{P C}$ & $668 / 700$ & 709 & 701 & 9 & 0.06 & 0.81 & 0.19 & 4.65 & 40 & 1.959 \\
\hline
\end{tabular}

MCD is used as an optical technique for the detection of the electronic structure of both ground and excited states (24). MCD can give information on the relative size of the $\triangle H O M O$ and $\triangle$ LUMO. Figure 1 shows the absorption and MCD spectra of compound $\mathbf{5}$ dissolved in DMSO. When the optical activity of compound $\mathbf{5}$ was investigated, a slight deviation was observed because its $\mathrm{Q}$ band showed less splitting, improved which this is attributed to its low symmetry as $C_{2 v}$ is isomer. The $B_{0}$ term appeared $+320 \mathrm{~nm}$ and $-370 \mathrm{~nm}$. Faraday $A$ term showed positive and negative sign since the excited state is orbitally degenerated. In the MCD spectrum, it was observed as a negative sign at $662 \mathrm{~nm}$ and $-691 \mathrm{~nm}$.

Figure 2 shows the absorption, emission, and excitation spectra of compound $\mathbf{5}$ in DMSO. The spectral data are listed in Table 1. Fluorescence emission peaks was observed at $709 \mathrm{~nm}$ for $\mathbf{5}$. The fluorescence quantum yield $\left(\Phi_{F}\right)$ value is 0.06 which is predicted for aggregated Pcs. Table 1 also shows $\Phi_{T}, \Phi_{d}, T_{F}$ and $T_{T}$ values of 5 . The high triplet state quantum yield value $\left(\Phi_{T}\right)$ is 0.81 . The $\Phi_{T}$ value suggests more efficient intersystem crossing (ISC), corresponding to low $\Phi_{\mathrm{F}}$ values. The values of compound 5 are 0.75 for ISC, 4.65 ns for fluorescence lifetime and $40 \mu$ s for triplet lifetime. A triplet decay curve of change in absorbance $(\Delta \mathrm{A})$ versus time in seconds is obtained from the experiment and from this the triplet lifetime can be determined as shown in Figure 3. All this data may indicate metal-free structure including aggregations.

Figure 4 shows spectral changes obtained during photolysis of complexes with increasing time from 0 to 140 seconds in the presence of DPBF and the results are summarized in Table 1 . DPBF degradation of the complexes 5 at $414 \mathrm{~nm}$ was monitored with UV-Vis spectrophotometry. There were no changes in the $\mathrm{Q}$ band intensities during these determinations, which is indicative of the fact that compound $\mathbf{5}$ is not degraded during singlet oxygen studies. The $\Phi_{\Delta}$ value is 0.19 . Although high triplet oxygen quantum yields of the phthalocyanine complex, accompany high singlet oxygen quantum yield, the compound gave a lower value of singlet oxygen quantum yield. This can be due to aggregation.

The photodegradation quantum yield value $\left(\Phi_{\mathrm{d}}\right)$ of compound $\mathbf{5}$ was obtained by decrease in the intensities of the $Q$ band under irradiation with increasing time from 0 to 60 minutes and the $\Phi_{d}$ was found as $1.959 \times 10^{-6}$. This result showed that the Pc is of high stability in DMSO (Figure 5). 


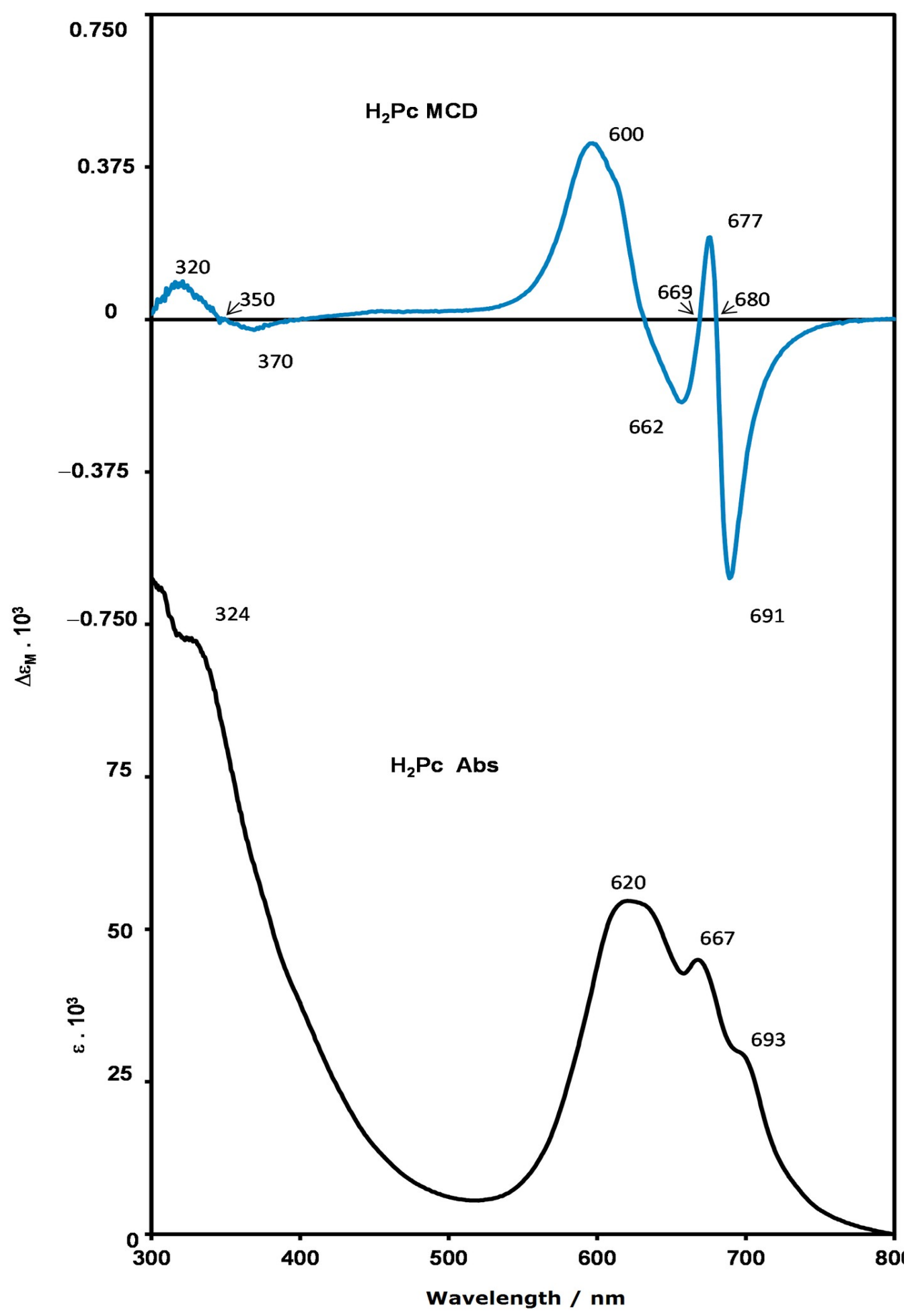

Figure 1. MCD (top) and UV-Vis (bottom) spectra for metal-free PC. 


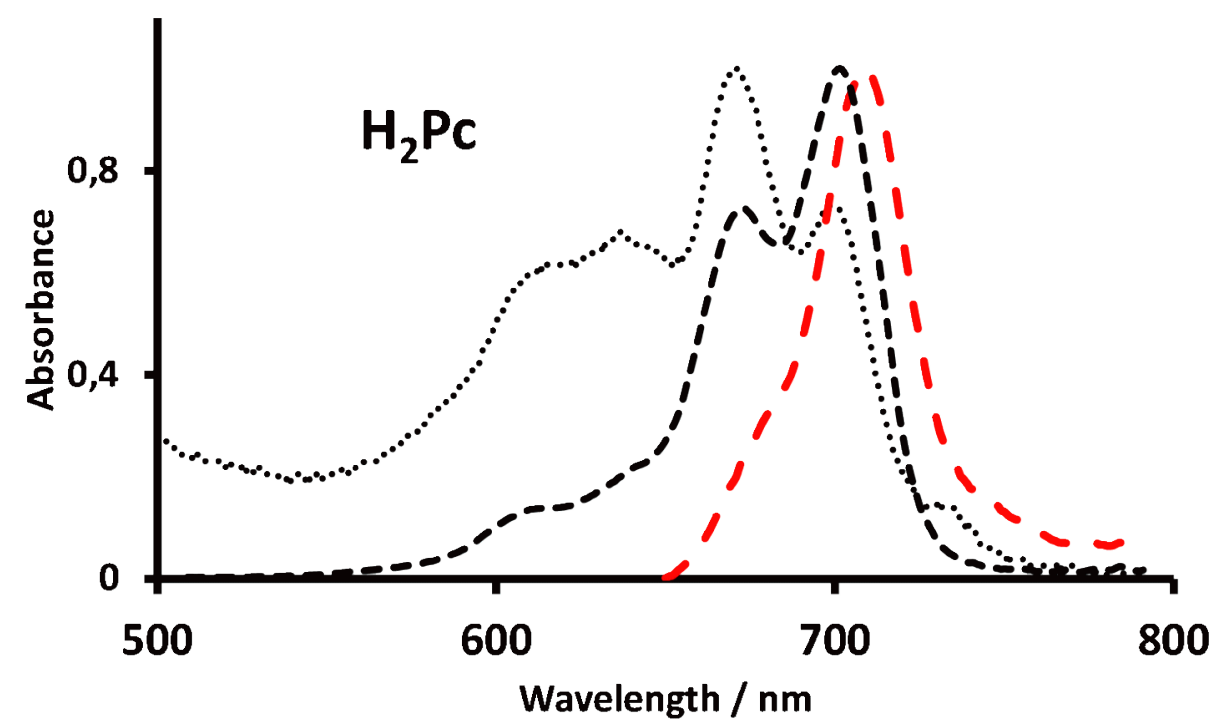

Figure 2. Absorption round dot line (black), excitation square dot line (black), and emission spectra dash line (red) of compound $5 \lambda_{\text {exc }}=606 \mathrm{~nm}$.

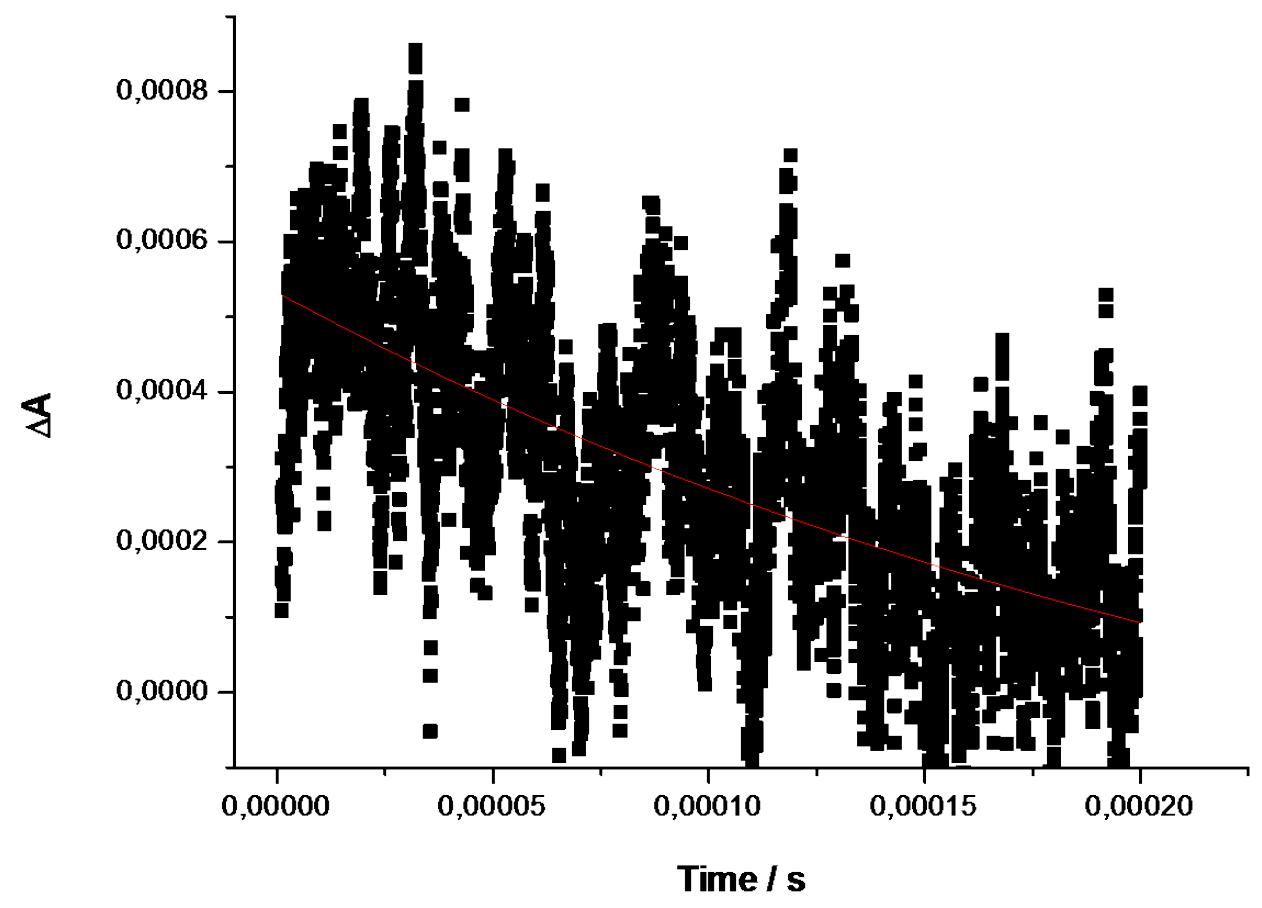

Figure 3. Triplet absorption decay curve for metal-free Pc. 


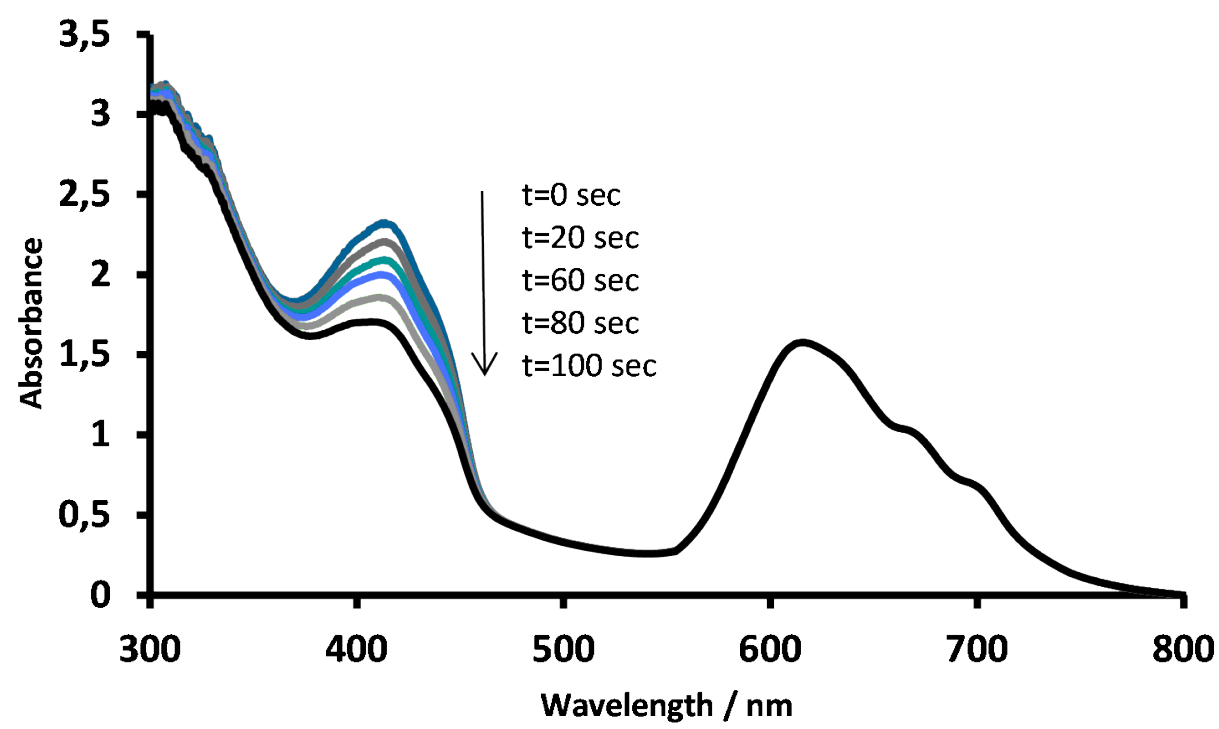

Figure 4. Time-dependent photobleaching of DPBF absorption in the presence of metal-free PC in DMSO.

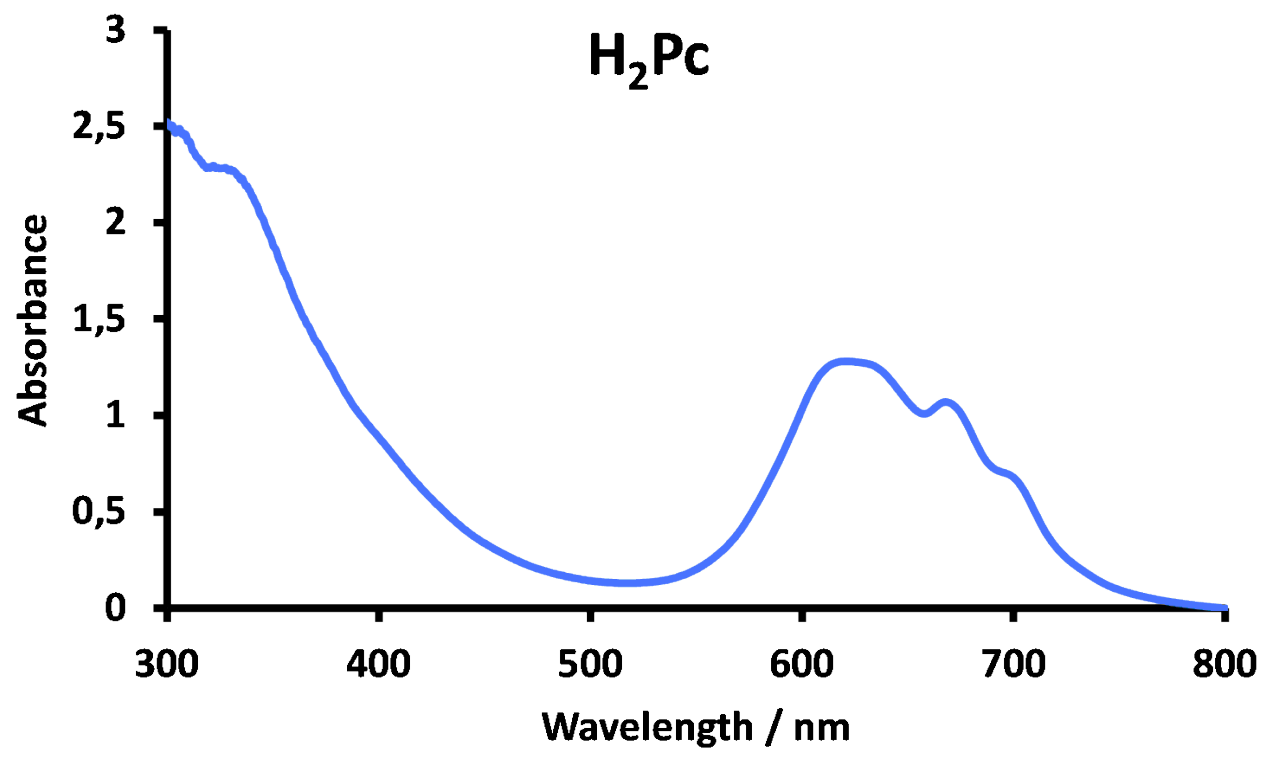

Figure 5: The spectra of photodegradation for metal-free Pc in DMSO.

\section{CONCLUSION}

$\beta$-substituted metal-free phthalocyanine which was obtained accidentally at $70^{\circ} \mathrm{C}$ in DMSO was obtained. The molecules have high photostabilities. The photophysicochemical behavior was found suitable for photodynamic therapy activity of the unmetallated phthalocyanine. The reaction is rapid enough to proceed are very low temperatures for phthalocyanine formation. Unfortunately, formation of ide products leads to low yields. The $\mathrm{Q}$ band of metal free Pc displays a slight deviation showing less splitting, attributed to lower symmetry as well as is also attributed to aggregation.

\section{ACKNOWLEDGEMENTS}

This work was supported by the Tubitak-BIDEB2219 International Postdoctoral Research Scholarship Programme, Applying Number: 1059B191401081 and the Research Fund of the Yildiz Technical University (Project No: 2012-0102-KAP12). The author is thankful to Prof. Kenneth K. Suslick and Prof. Tebello Nyokong for providing me the grant and the facilities in the lab during the research. 


\section{REFERENCES}

1. Braun A, Tcherniac J. Uber die Producte der Einwirkung von Acetanhydrid auf Phatalamid, Ber. Deutsch. Chem. Ges., 40, 1907; 40: 2709- 2714.

2. Leznoff CC, Lever ABP. (Eds.),Phthalocyanines: Properties and applications, C.C. VCH Publishers, New York, (1989, 1993, 1993, 1996); 1-4.

3. Kadish KM, Smith KM, Guilard R. (Eds.), The Porphyrin Handbook: Applications of Phthalocyanines, The Porphyrin Handbook, Academic Press, New York 2000; 19.

4. de la Torre G, Vásquez P, Agulló-López F, Torres T. Role of structural factors in the nonlinear optical properties of phthalocyanines and related compounds. Chem. Rev. 2004; 104: 3723.

5. Rella R, Serra A, Siciliano P, Tepore A, Troisi L, Valli L. Characterization of novel copper phthalocyanine Langmuir-Blodgett-films for $\mathrm{NO}_{2}$ detection. Thin Solid Films. 1996; 284-285:870.

6. Armund F, Ferez H, Fouriaux S, Araspin O, Pradeau JP, Claessens CG, Maya EM, Vásquez $P$, Torres $T$. Tetraamidometallo-phthalocyanines Langmuir-Blodgett films: Morphology versus central metal effects on $\mathrm{NO}_{2}$ detection. Synthetic Metals. 1999; 102: 1476.

7. Snow AW, Barger WR, Klusty M, Wohltjen $H$, Jarvis NL. Simultaneous electrical conductivity and piezoelectric mass measurements on iodine-doped phthalocyanine Langmuir-Blodgett films. Langmuir 1986; 2: 513.

8. Rosenthal I. Phthalocyanines as photodynamic sensitizers. Photochem. Photobiol. 1991; 53: 859.

9. Spikes JD. New trends in photobiology: Chlorins as photosensitizers in biology and medicine. J. Photochem. Photobiol. B 1990:6: 259.

10. Brown SG, Tralau CJ, Coleridge-Smith PD, Akdemir DT, Wieman TJ. Photodynamic therapy with porphyrin and phthalocyanine sensitisation: quantitative studies in normal rat liver. Br. J. Cancer. 1986; 54: 43.

11. Bonnett $\mathrm{R}$, in Chemical Aspects of Photodynamic Therapy, D. Phillips (Ed.),Gordon and Breach Science, Canada, 2000.

12. Wöhrle D, Suvorova O, Gerdes R, Bartels O, Lapok L, Baziakina N, Makarov S, Slodek A. Efficient oxidations and photooxidations with molecular oxygen using metal phthalocyanines as catalysts and photocatalysts. J. Porphyrins Phthalocyanines. 2004; 8: 1020.
13. Mele G, Sole RD, Vasapollo G, García-López E, Palmisano L, Schiavello MJ. Photocatalytic degradation of 4-nitrophenol in aqueous suspension by using polycrystalline TiO2 impregnated with functionalized Cu(II) -porphyrin or Cu(II)-phthalocyanine. Catalysis. 2003; 217: 334 .

14. Sehlotho N, Nyokong T. Catalytic activity of iron and cobalt phthalocyanine complexes towards the oxidation of cyclohexene using tert-butylhydroperoxide and chloroperoxybenzoic acid. J. Mol. Catal. A: Chem. 2004; 209: 51 .

15. Tolbin AY, Ivanov AV, Tomilova LG, Zefirov NS. Preparation of 1,2-bis(3,4dicyanophenoxymethyl)benzene and the binuclear zinc phthalocyanine derived from it. Mendeleev Commun. 2002; 12: 96 .

16. Tolbin AY, Ivanov AV, Tomilova LG, Zefirov NS. Synthesis of 1,2-bis(3,4-dicyanophenoxymethyl)benzene and binuclear zinc phthalocyanines of clamshell and ball types. J Porphyr. Phtalocyan. 2003; 7: 162.

17. Canlica M. Photo-physico-chemical properties of 1,3benzenediol-substituted face-to-face Phthalocyanines. Inorganica Chimica Acta 2020; 511: 119830.

18. Ali H, Van Lier JE. Rhodium-catalyzed modification of Phthalocyanines. Tetrahedron Lett. 2013; 54: 2956.

19. Canlica M. Synthesis, photophysics, and photochemistry of ball-type phthalocyanines. J. Photochem. Photobiol. A Chem. 2019; 384: 112043.

20. Canlica M, Omur BC, Salih B. Synthesis, photophysical, photochemical and $\mathrm{SO} 2$ sensing properties of ball-type phthalocyanines substituted with carboxyl groups. Inorg. Chem. Comm. 2019; 103: 75.

21. Greenberg S. Lever ABP, Lenoff CC. Approaches towards the synthesis of a 2,9,16,23-tetrasubstituted phthalocyanine as a pure isomer. Can. J. Chem. 1988; 66: 1059.

22. Keller TM, Dominguez DD. High temperature resorcinol-based phthalonitrile polymer. Polymer. 2005; 46: 4614 .

23. Kobayashi N. Lever ABP. Cation or solvent-induced supermolecular phthalocyanine formation: crown ether substituted phthalocyanines. J. Am. Chem. Soc. 1987; 109: 7433.

24. Kobayashi N, Nakai K. Applications of magnetic circular dichroism spectroscopy to porphyrins and Phthalocyanines. Chem. Commun. 2007; 4077. 
Canlica M. JOTCSA. 2020; 7(3): 875-882.

RESEARCH ARTICLE 